\title{
Mobile Tourist Guide - An Intelligent Wireless System to Improve Tourism, using Semantic Web
}

\author{
$\underline{\text { http://dx.doi.org/ijim.v5i4.1695 }}$ \\ Hosam F. El-Sofany ${ }^{1}$, and Samir A. El-Seoud ${ }^{2}$ \\ Cairo Higher Institute for Engineering, Computer Science, and Management, Cairo, Egypt \\ Technische Universität Darmstadt (TU Darmstadt), Darmstadt, Germany
}

\begin{abstract}
With the recent advances in Internet and mobile technologies, there are increasing demands for electronic access to tourist information systems for service coordination and process integration. Mobile computing and mobile devices are used to implement various tourist services (e.g. electronic tourist guides, digital interactive maps, and tourist e-commerce transactions). However, due to disparate tourist information and service resources such as airlines, hotels, tour operators, it is still difficult for tourists to use them effectively during their trips or even in the planning stage. Neither can current tourist portals assist tourists proactively. To overcome this problem, we propose the analysis, design, and implementation of the "Mobile tourist guide" system, that access through wireless devices and use Semantic Web technologies for effective organization of information resources and service processes. The proposed system provides the users with various services such as: 1) displaying the shortest path between the sources and destinations the visitors specify, 2) displaying general information of shops, newest events of the plaza and shops, 3) provides service of hotel, restaurant and cinema-ticket reservations, 4) provides user-friendly administration service. The Admin can manage the position, blocking path details, general information of hotel, restaurant, shops and plaza, and reservation details via web browser without changing the framework of the system. The system prototype has been developed on the top of Java 2 Micro Edition which offers an ideal platform for the development of full-fledged, interactive and portable applications tailored for resource constrained mobile devices. The paper presents our development experiences and highlights its main advantages and limitations in relation to the implementation of such kind of applications.
\end{abstract}

Index Terms-Electronic tourist guide, Mobile tourist guide, Semantic Web technology, Tourist Information System, Tourist application.

\section{INTRODUCTION}

Tourism is a leading industry in the e-business. There are several reasons behind this fact. Tourism is an information-intensive industry with a long value chain. Since 1960s this industry represents a classical field of information and communication technology applications. Characteristics of electronic tourism marketplace lead to many challenges. One of them is growing need for interoperability between information systems, allowing seamless information exchange between tourism organizations. The traditional solution for interoperability issues was to develop interfaces between each pair of communication sys- tems, but development and maintenance of such programs are too expensive and not flexible enough for the electronic tourism marketplace. There have been many attempts to standardize data exchange within tourism electronic markets in order to reach global interoperability. As a result there are some global standards used only by large companies and some standards developed for the needs of small companies, but used only on national level [1].

The World Tourism Organization predicts that by 2020, tourist arrivals around the world will increase over 200\%. Tourism has become a highly competitive business all over the world. Competitive advantage is increasingly driven by the advancement of information technology and innovation. Currently, the Internet is the primary source of tourist destination information for travelers.

With the recent advances in hardware and software technologies, the Internet is quickly evolving towards wireless adoption. New mobile applications running on these devices provide users with easy access to remote services available anytime and anywhere, and will soon take advantage of the ubiquity of wireless networking in order to create new virtual worlds. Besides, intelligent software agents can run on these devices and can provide personalized assistance to tourists during their trip. Together with traditional information agents such as hotel broker agents, tour planning agents, and other disparate tourist resources, they form a Multi-Agent Information System, for collaborative and intelligent assistance to tourists.

At the same time, Semantic Web technologies have been maturing to make e-commerce interactions more flexible and automated. Ontology defines the terms used to present a domain of knowledge that is shared by people, databases, and applications. In particular, ontology encodes knowledge, possibly spanning different domains as well as describes the relationships among them. Currently, ontology is actively being developed in various business domains. The Semantic Web provides explicit meaning to the information available on the Web for automated processing and information integration based on the underlying ontology. Our aim in this project is to describe characteristics of the electronic tourism as well as challenges related to the tourism electronic marketplace and to discuss how semantic web technologies can help to resolve these challenges.

In this research, we intend to expand tourist coordination and integration towards complete support by employing all the above-mentioned technologies. The main challenge of our system is to provide an effective coordination and integration of disparate information and service re- 
sources anytime, anywhere, as well as the provision of personalized assistance and automation to the tourists, each having different preferences and support requirements that often being changed during the trip. With the help of ontology, our system can help tourists better understand and guide them to specify their needs and preferences collaboratively, so that the appropriate information and services resources could be located from the Semantic Web.

Because scalability and flexibility, tourists cannot be flexibly assisted in a centralized manner. The assistance of increasingly powerful mobile devices becomes the enabling technologies. Under individual's instructions and preferences, intelligent software agents within the system can be delegated to help recommend, plan, and negotiate personalized activities and schedules, thereby augmenting the user's decisions collaboratively. We introduce a scalable, flexible, intelligent, and multi-agent "Mobile tourist guide" system, with agent clusters for tourist service coordination and integration. Each agent cluster comprises several types of agents to achieve the goals of the major tasks of a tourist's trip, such as, information gathering, preference matchmaking, planning, service brokering, commuting, and mobile servicing. The agents also make use of ontology from the Semantic Web to search information and make recommendations to the tourists. Further, we detail how this can be effectively implemented with Web service and Semantic Web technologies, integrating disparate Internet tourist resources

The research goal is to analysis, design, and implement the "tourist guide" system, that access through wireless devices (such as mobile telephones, Personal Digital Assistants - PDAs, and laptops). The following are some services and functionality provides by the proposed research study:

- The system provides general information of hotel, restaurant, shops, hospitals, and companies. As well as the newest events of the plaza and shops.

- The system provides service of hotel, restaurant and cinema-ticket reservations.

- The system provides service of displaying the shortest path between the sources and destinations the visitors specify.

- The system provides user-friendly administration service. The Admin can manage the position, blocking path details, general information of shops and plaza, and reservation details via web browser without changing the framework of the system.

- The system tackles the difficulties of displaying the routes on different floors and many problems in mobile web development.

\section{SySTEM OVERVIEW}

The Semantic Web as the next generation web is the vision of having background knowledge about the meaning of web sources stored in a machine-processable and interpretable way. The area of tourism is highly dynamic area that currently already extensively uses the available Internet technologies. However, the shortcomings of the existing technology are that information finding and extraction as well as the interpretation of the information contained in the web sources is left to the human user. The management and interoperation of semantically diverse tourism information systems are facilitated by Semantic Web technology that provides methods and standards, which allow accurate access to information as well as flexibility to comply with needs of tourism information system users and administrators. In this research project we introduce a new Semantic Web framework that can enable knowledge sharing and reuse. The Semantic Web uses agent technologies to formally model information represented in web resources. This makes it accessible to humans and computers working together, perhaps in conjunction with intelligent network services such as search agents.

The "Mobil tourist guide" system represents a relatively new trend in the field of tourism and involves the use of Semantic Web technology and mobile devices as electronic tourist guides. While much of the underlying technology is already available, there are still open challenges with respect to design, usability, portability, functionality, and implementation aspects. This research study presents the analysis, design and implementation of the "Mobil tourist guide" system, that allows the users to download these personalized applications either directly to their mobile device or first to a PC and then to a mobile terminal (through infrared or Bluetooth). Thereafter, network coverage is not further required as the applications execute in standalone mode and may be updated when the user returns online. The system prototype will use Java 2ME, which offers an ideal platform for the development of fullfledged, interactive and portable applications tailored for resource constrained mobile devices.

In our research study we consider number of important issues related to the Semantic Web that belongs to four categories: Semantic Web languages, ontologies, semantic markup of Web pages, and Semantic Web services [2].

Semantic Web Languages: In order to represent information on the Semantic Web and simultaneously make that information both syntactically and semantically interoperable across applications, it is necessary to use specific languages. There are a lot of such languages around, and most of them are based on XML, XML Schemas, RDF, and RDF Schemas, all four developed under the auspices of W3C and using XML syntax.

Ontologies: Ontology comprises a set of knowledge terms, including the vocabulary, the semantic interconnections, and some simple rules of inference and logic for some particular topic. Ontologies applied to the Web are creating the Semantic Web. Ontologies provide the necessary armature around which knowledge bases should be built, and set grounds for developing reusable Webcontents, Web-services, and application. Ontologies facilitate knowledge sharing and reuse, i.e. a common understanding of various contents that reaches across people and applications.

Semantic Markup: Ontologies merely serve to standardize and provide interpretations for Web content, but are not enough to build the Semantic Web. To make Web content machine-understandable, Web pages and documents themselves must contain semantic markup, i.e. annotations which use the terminology that one or more ontologies define and contain pointers to the network of ontologies.

Semantic Web Services: Intelligent, high-level services like information brokers, search agents, information filters, intelligent information integration, and knowledge 
management, are what the users want from the Semantic Web. They are possible only if a number of ontologies populate the Web, enabling semantic interoperation between the agents and the applications on the Semantic Web, i.e. semantic mappings between terms within the data, which requires content analysis.

\section{A. Semantic Web and Tourism Information Systems}

The Semantic Web appears as a promising technology for implementing E-tourism information system. The Semantic Web constitutes an environment in which human and machine agents will communicate on a semantic basis [8]. One of its primary characteristics, viz. shared understanding, is based on ontologies as its key backbone. Ontologies enable the organization of tourism objects around small pieces of semantically annotated tourism objects [9]. Items can be easily organized into customized tourism services and delivered on demand to the user, according to her/his profile and business needs.

The term "Semantic Web" encompasses efforts to build a new WWW architecture that enhances content with formal semantics. That means, content is made suitable for machine consumption, as opposed to content that is only intended for human consumption. This will enable automated agents to reason about Web content, and produce an intelligent response to unforeseen situations.

"Expressing meaning" is the main task of the Semantic Web. In order to achieve that objective several layers of representational structures are needed. They are presented in the Figure 1, among which the following layers are the basic ones [8]:

- The XML layer, which represents the structure of data,

- The RDF layer, which represents the meaning of data,

- The Ontology layer, which represents the formal common agreement about meaning of data,

- The Logic layer, which enables intelligent reasoning with meaningful data.

The important property of the Semantic Web architecture (i.e., common-shared-meaning and machineprocessable metadata), enabled by a set of suitable agents, establishes a powerful approach to satisfy the E-tourism requirements. The process is based on semantic querying and navigation through tourist services, enabled by the ontological background. The Semantic Web can be exploited as a very suitable platform for implementing an Etourism information system, because it provides all means for electronic tourism: ontology development, ontologybased annotation of tourism objects, their composition in tourism recourses and active delivery of the tourism services through E-tourism portals.

The main style related to our research in the Semantic Web is the layered architecture. The layered architecture consists of a number of layers organized hierarchically as in Figure 1. Each layer provides a service to its upper layer and serves as a client to the layer below. This kind of architecture depends on the sequence of incremental steps, which means the increasing level of abstraction; if the function of one layer changes, this means that two other layers may be effected.

In the tourism industry, the Semantic Web technologies offer considerable benefits in terms of travel management,

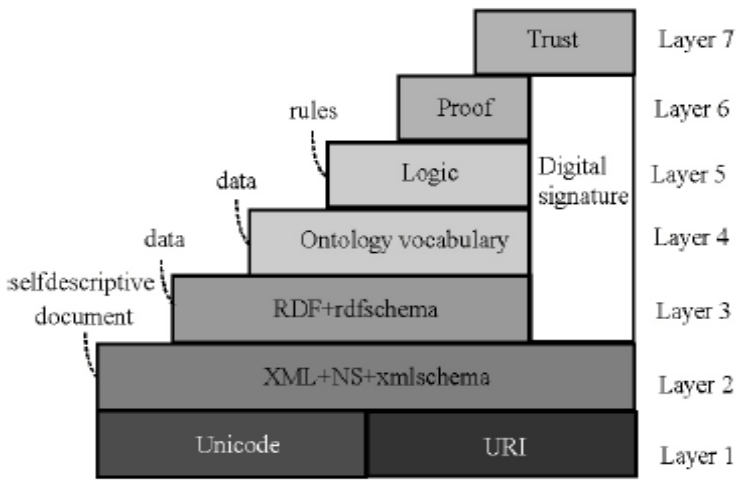

Figure 1. Layers of the Semantic Web

content and document management, knowledge management, supply chain management and integration of distributed applications and services [16,17]. The Semantic Web technologies help in the realization of the vision of the intelligent wireless Web in the following ways: Deeper understanding of the semantics of document content and travel task structure using ontologies, will help the mobile tourist in intelligent information retrieval, extraction and processing, thereby helping him to accomplish elements of a travel plan. Through the introduction of ontological reasoning, Semantic Web techniques are suitable for flexibly discovering abilities in using information that was not specifically designed or intended for a particular use case. Given the dynamic nature of travel processes, this would help in on-the fly resource discovery and integration. Mobile tourists will be able to dynamically locate highly specific data and services on an asneeded basis [18].

Travel enterprises very often undertake their processes in different ways. Differences in the meanings of terms make collaboration difficult. The use of shared ontologies and semantic standards will ensure increased interoperability across devices, platforms and applications [19].

Separation of presentation and data, as ensured by the Semantic Web technologies, will ensure the use of the same middleware tier for both mobile and fixed network clients. The Semantic Web technologies can provide a standardized way to interpreting context, enabling both human and software agents to infer new context knowledge and consequently take intelligent actions.

\section{B. System Architecture}

Our basic Semantic Web framework illustrated in Figure 2, provides the tourist with different types of tourism services. In this research study we focus on the implementation of these services that provide the tourist with the following services:

- General information of hotel, restaurant, shops, hospitals, and companies

- $\quad$ The newest events of the plaza and shops.

- Hotel, restaurant and cinema-ticket reservations.

- displaying the shortest path between the sources and destinations the visitors specify, and

- Semantic search.

On the other hand, the system allows the administrators to manage the position, blocking path details, to purplish the general information of shops and plaza, and reservation details via web browser. 


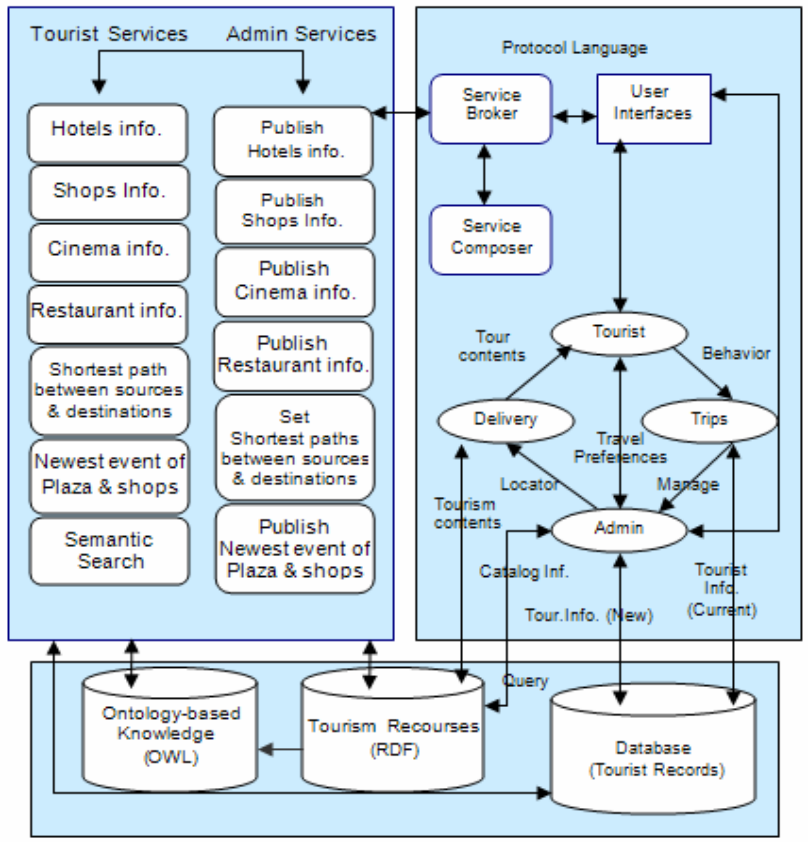

Figure 2. Basic Semantic Web Model for Tourist Guide System

\section{Using Mobile technology for E-Tourism}

M-tourism means the provision of tourism services on wireless devices: portable computers, PDA's, mobile telephones and Tablet PC. Our mobile tourist guide system has functions to enter and to organize the tourism objects in the database and to make them available for tourists and administrators using the E-tourism management information systems (TMIS) portals in which the tourism process and its activities have been structured. The M-tourism object management systems are now compatible for all kind of user devices, the conventional desktop PC as well as the mobile devices laptop, PDA and mobile telephone.

- The advantages: The advantages of mobile tourism refer mostly to the mobile user: a great flexibility, an improved tourist schedule is possible, increased productivity during dead moments and just in time tourism.

- Difficulties and limitations: Nevertheless they have very small screens, limited memory capacity and the large diversity of mobile devices obstruct a good tourism experience. In the M-tourism field we notice that the tourism services must answer to specific conditions. We will have to get around the technical restrictions so we can create a good tourism services. The usage of video, audio, clear interfaces and divided tourism services must contribute to this. Furthermore we will have to adapt the content to the needs of the mobile user. Because the user has a fragmentized time schedule, we will have to be sure that the tourism objects are not to long. Dividing the knowledge in smaller modules offers a solution.

\section{AnAlysis AND DESIGN MEthods}

We build first the general architecture of the system, set the graphical user interface and design the structure of the database that stores the required data and information. In order to achieve our goals we defined the following tasks:

\section{A. System Analysis}

The analysis phase is the main phase in which the assessment system requirements are identified in more details. The goal of this phase in the system development is to represent the system goals into defined functions and operations of the intended applications. The tourist guide system requirements are documented in a complementary set of artifacts (flowcharts, use case diagrams, system sequence diagrams...). Each artifact provides a different perspective of the system under design and contains distinct requirements. The combination of these perspectives establishes the tasks that are to be accomplished by the system.

- Information Gathering Techniques: By assisting of some specialist employees from different tourism companies, all the system services and materials are checked. The following are some information gathering techniques that used in the tourist guide system analysis.

- Interviews: The most common technique for gathering requirements is to sit down with the clients (tourists, tourism companies, and administrators) and ask them what they need. Our discussion would help us plan out ahead of time, based on the type of requirements that we are looking for.

- Prototyping: We use this technique for gathering requirements. In this approach, we gather preliminary data and requirements that we use to build the initial version of the system.

- $\quad$ UML (Universal Modeling Language) use-cases is used also in the analysis of the tourist guide system as a model tool, in which the functional requirements are extracted and described along with a scenario of the flow of events.

The system requirements are classified in two types: functional and non-functional requirements.

- Functional requirements: The tourist guide system allows different types of users to interact with the system according to given privileges. There are three main users of the system: administrator, tourism company, and tourist, as shown in Figure 3. The system provides the following applications (services) to each user:

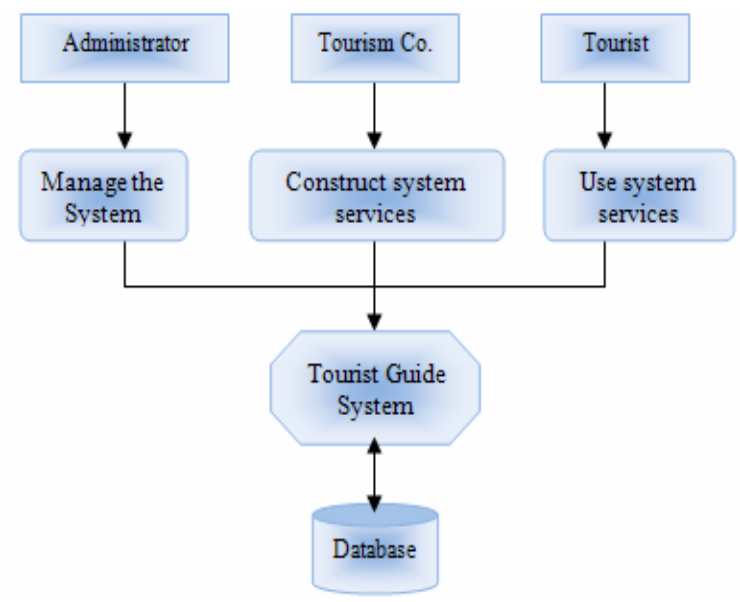

Figure 3. The main users of the system 
1. The administrator application: This application provides the tourism administrator with simple tools to manage the information of the tourist guide system. The following are some features provided by the administrator application:

- Activate/inactivate the system.

- Import and export the system' database

- Send automatic email immediately containing the new password whenever the user changes his/her password.

- Administer the basic data and information of the system: hotels, restaurant, shops, hospitals, companies, and etc.

2. The tourism company application: This application provides the tourism companies with various tools to construct the system services. The following are some features provided by the tourism company application:

- Change account password

- Manage (add, update, delete and display) all the tourist guide system services.

3. The tourist application: This application provides the tourist with interactive tools (web-based and mobilebased) to use the system services. The following are some features provided by the tourist application:

- Create (and change) account password

- Search for information of hotel, restaurant, shops, hospitals, and companies.

- $\quad$ Search for the newest events of the plaza and shops.

- Hotel, restaurant and cinema-ticket reservations.

- displaying the shortest path between the sources and destinations the visitors specify.

- Access the tourist guide system through wireless devices (such as, Mobile phone, PDA and Tablet PC) and take quick tour.

- Non-functional requirements: In this part, we have verified and proved the system' aspects and interfaces that are not directly related to the functional behavior, such as: usability, reliability, supportability, performance, and security:

- Usability: Is the system providing a coherent and flexible user interface that can be used easily with all users? Has the system an obvious component which allows the user to navigate easily? Are the system applications easy to use by tourism companies and tourists? Is the system adaptive to user needs? Is the tracking component working properly and produce the expected results

- Supportability: Can the system be easily modified or extended easily? Can tourism companies and administrators add materials easily?

- Contents and Design: Are the design and contents of the system satisfactory to tourists, tourism companies, and system administrators? What are their feedbacks? Does the system cover well the topics? Are they useful and meet their goals? Quality of texts, images, sounds, and clips? We will compare the progress of tourists.

- Performance: Are the performance requirement concerned with quantifiable attributes of the system, such as response time, throughput availability and accuracy?

- Flexibility: Is the system flexible, since it will be used regularly with a new set of initial data and can accommodate changes in database such as modifying the contents?

- Security: Is the system preventing unauthorized users to access the system?

- Hardware and software resource: We have used some software during our first prototype of the system development such as: Windows XP professional, Windows Server O/S, Microsoft office Excel, Microsoft Word, SQL Server database system, MS Visual Studio.Net, and Photo Shop.

To extend our research work, by using Semantic Web and Mobile technologies, we used other new languages and tools including: XML- eXtensible Markup Language, XML Schemas, RDF- Resource Definition Framework, RDF Schemas, OWL- Web Ontology Language, and Java 2ME.

\section{B. System Design}

Any successful web-based system should have an easy to use graphical user interface (GUI) that facilitates its use and flexibility. Thus the proposed system should possess an attractive GUI that is suitable to the potential users. The interface should be tested by its users and updated whenever necessary.

The tourist guide system interface is entirely webbased, and doesn't necessitate any technical skills from the potential users. The system is appropriate for webbased search, with access to the Internet, or mobile-based search using the wireless devices.

- Database design: Database is an important component in our system. We have used Microsoft SQL Server database as a database management system (DBMS). The DBMS stores the following entities:

- Tourist (name, id, nationality, tel, email, ...)

- accompany (name, id, relation, ...)

- Hotel (name, tel, add, ...)

- Hospital (name, tel, add, ...)

- Shops (name, tel, email, add, ...)

- Restaurants (name, tel, email, add, ...)

- Companies (name, tel, email, add, ...)

- Conferences (name, type, location, start_date, end-date, ...)

- Services (Sno, type,...)

- Travel_agent (id, name, tel, fax, email, add, ...)

- Tours (location, arrive_time, pickup_time, location, ...)

- Admin (id, name, tel, email, ...).

We have chosen the entity-relationship model to represent the database design. As shown in Figure 4, we present the initial entity-relationship diagram of the tourist guide system, that contains the main entities (tables) used for creating the system database. 


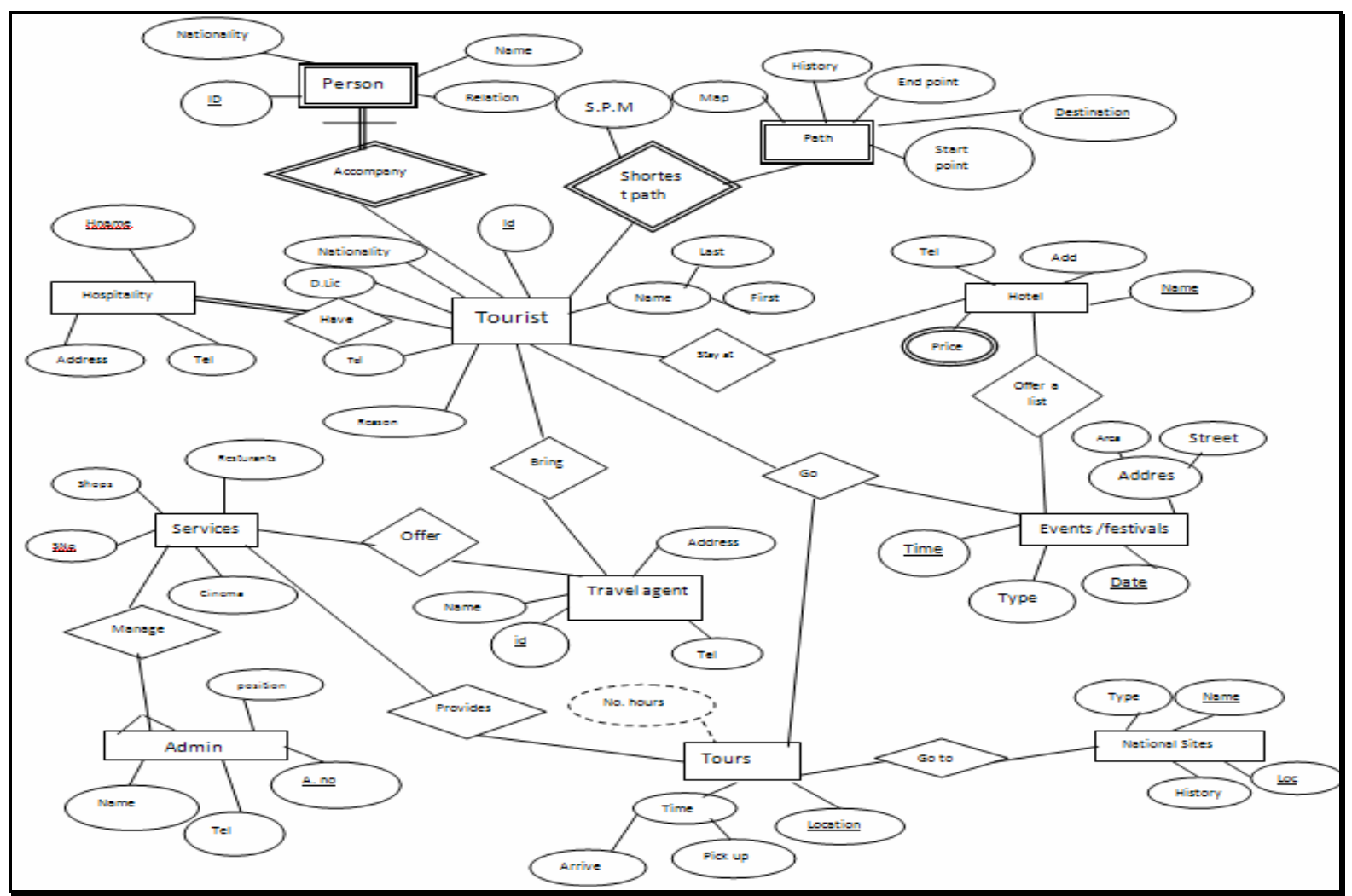

Figure 4. Basic entity relationship diagram

\section{A. Advantages of the System}

The proposed research study is of considerable significance due to the following facts:

- The new generation of the Web, the so-called Semantic Web, appears as a promising technology for implementing E-tourism systems.

- E-tourism and M-tourism (Electronic and Mobile tourism) research areas can benefit from Semantic Web technologies. The Semantic Web technology has enabled by a set of suitable agents, which seems to be powerful enough to satisfy the E-tourism requirements fast, and just-in-time.

- The Semantic Web has opened new horizons for internet applications in general and for E-tourism in particular.

- It is extremely important for Tourism Authority to improve the tourism services, thus promoting significant changes among tourism institutions.

- The study is significant to the researcher who will be working on open, hot and recent research areas of the next generation Web.

- The study is significant to researchers who will be working on building Web-based system using new techniques (such as; Semantic Web, and Mobile technology) and new languages and tools (such as; $X M L$ eXtensible Markup Language, XML Schemas, RDFResource Definition Framework, RDF Schemas, $O W L$ - Web Ontology Language, and Java 2ME ).

- The study allows the (new) employees and workers to use his/her wireless devices (PDA, Mobile telephone, and Smart phones) anytime and anywhere to know the places that they are looking for easily and quickly.
- Tourists, especially first time tourists, will feel comfortable. This is because all what they need to know about national sights; restaurants and other interesting places will be in front of them by pressing a few buttons.

- $\quad$ Tourists will be able to save time by knowing the information they need without having to call or spend time on the internet searching.

- $\quad$ First time drivers, or drivers who came from abroad will be able to find their way quickly and smoothly.

- $\quad$ The hotels, hospitals, companies and other important sectors will not receive unnecessary calls like calls that ask about the building's site or departments' phone numbers, etc. This is because this kind of information will be available on the proposed system.

- The system guides the researchers and guests that came for conferences to spend an easy tour in the country.

- The system guides the visitors that came from outside for festivals and Eid period, for places where celebration is, without wasting their time.

\section{CONCLUSION AND FUTURE WORK}

In this paper, we have presented the architectural details and design for "Mobile tourist guide" system, that used Semantic Web technologies for effective organization of information resources and service processes. The proposed system allows the users to: display general information of hotels, restaurant, shops, hospitals, companies, etc; display the shortest path between the sources and destinations the visitors specify; the newest events of the plaza and shops; provide service of hotel, restaurant and cinema-ticket reservations; and allow the administrators to 
manage the position, blocking path details, general information of hotel, restaurant, shops and plaza, and reservation details via web browser without changing the framework of the system. The system prototype has been developed on the top of Java 2 Micro Edition which offers an ideal platform for the development of full-fledged, interactive and portable applications tailored for resource constrained mobile devices. The paper presented our vision of the Semantic Web and its potential benefits to tourism information systems. In tourism much data is only loosely structured or given in texts - a mode that is too weak for rich querying. The Semantic Web provides additional mechanisms adequate for dealing with these structures, enabling new applications for commerce and communications, especially. We have proposed a Semantic web framework that supports multiple platforms (in particular wireless mobile ones) and a methodology for the analysis and design of Mobile tourist guide system.

As for future research, our key focus is about thr implementation of "Mobile tourist guide" ontologies. Despite the considerable efforts toward the construction our system ontologies for agent interoperation, some difficulties and challenges are still open. Throughout the paper, we assume ontologies to be consistent and available to the agents. In reality, many ontologies are redundant in the sense that they present the same domain with little or no interoperation between different ontologies. The ontologies play a main role in the system implementation, as they provide a shared representation of the domain and of the concept that the agents need to use.

\section{REFERENCES}

[1] Egypt Authority Web site: http://sharepoint.emot.gov.eg

[2] Fayed Ghaleb, Sameh Daoud, Ahmad Hasna, Jihad Jaam, Samir A. El-Seoud, and Hosam El-Sofany, "E-Learning Model Based On Semantic Web Technology". International Journal of Computing \& Information Sciences (IJCIS), Vol. 4, No. 2, P63-71, August 2006.

[3] Berners-Lee, T., “Semantic Web on XML," Dec.2000; http://www.w3.org/2000/ Talks/1206- xml2k-tbl/

[4] C Cody Burleson. Introduction to the Semantic Web Vision and Technologies. http://www.semanticfocus.com/blog/entry/title/ introduction-to-the-semantic-web-vision-andtechnologies-part-2foundations/, 2007.

[5] Matthews, B.,"Semantic Web Technologies. JISC Technology and Standards Watch,” http://www.scribd.com/doc/300024/What-isweb- 20-Ideas-technologies-and-implications-Paul- Anderson, 2005.

[6] Davis, M., Moving to Unicode 5.1. http://googleblog.blogspot .com /2008/05/movingto- unicode-51.html, 2008.

[7] Berners-Lee, T., Uniform Resource Identifiers, URI Generic Syntax. IETF. <http://www.ietf.org/rfc/rfc2396.txt>, 2006.

[8] Patel-Schneider, P.F. and D. Fensel. Layering the Semantic Web: Problems and Directions. In the Proceeding of 1st International
Semantic Web Conference (ISWC 2002). Sardinia, Italy, 9-12 June, pp: 476, 2002.

[9] Buraga, S. and G. Ciobanu. A RDF- based model for expressing spatio-temporal relation between web sites. In The 3rd International Conference on Information Systems Engineering. IEEE Computer Society. pp: 355. IEEE Computer Society Washington, DC, USA, 2002.

[10] Miller, E., "An Introduction to the Resource Description Framework", in D-Lib Magazine, May.1998; <http://www.dlib.org/ dlib/may98/miller/ 05miller.html>.

[11] Nejd, W., D. Olmedillal and M. Winslett. Peer Trust: Automated Trust Negotiation for Peers on the Semantic Web. Lecture Notes in Computer Science: Secure Data Management. Springer Berlin/Heidelberg, vol.3178/2004. pp: 118-132., 2004.

[12] Matthew Richardson , Rakesh Agrawal and Pedro Domingos, et al. Trust management for the semantic web. Lecture Notes Comput. Sci., 2870: 351-368, 2003. http://dx.doi.org/10.1007/978-3540-39718-2_23

[13] Brian Matthews and Theo Dimitrakos., "Deploying Trust Policies on the Semantic Web, <http://epubs.stfc.ac.uk/bitstream/ 638/SWADtrust2004.pdf>, 2004.

[14] Russell Cloran and Barry Irwin. XML Digital Signature and RDF. http://icsa.cs.up.ac.za/issa/2005/Proceedings/Poster/026 Article.p df, 2005.

[15] Mark Bartel, John Boyer, Barb Fox and Brain La Macchia, et al. XML Signature Syntax and Processing. <http://www.w3.org/ TR/xmldsigcore/>.

[16] Kanellopoulos, D., Kotsiantis, S., Pintelas P. Intelligent Knowledge Management for the Travel Domain. GESTS International Transactions on Computer Science and Engineering, Vol. 30, No.1, pp. 95-106, 2006.

[17] Dimitris K. and Sotiris K., "Towards Intelligent Wireless Web Services for Tourism". IJCSNS International Journal of Computer Science and Network Security, VOL.6 No.7B, July 2006.

[18] Lassila, O., Adler M. Semantic gadgets: ubiquitous computing meets the semantic web. In: D. Fensel, J.A. Hendler, H. Lieberman, W. Wahlster (Eds.) Spinning the Semantic Web: Bringing the World Wide Web to Its Full Potential, MIT Press, 2003, pp. 363- 376, 2003.

[19] Missikoff, M., Werthner, H. Höpken, W., Dell'Ebra, M., Fodor, O. Formica, A., Francesco, T. HARMONISE: Towards Interoperability in the Tourism Domain. Proceedings of Information and Communication Technologies in Tourism 2003, ENTER 2003, pp. 58-66, Helsinki: Springer, 2003.

\section{AUTHORS}

Hosam F. El-Sofany is with Cairo Higher Institute for Engineering, Computer Science, and Management, Cairo, Egypt

Samir A. El-Seoud is with Technische Universität Darmstadt (TU Darmstadt), Darmstadt, Germany.

This article is an extended version of a paper presented at the International Conference on Computer Aided Learning (ICL2010), held at Hasselt University, Hasselt, Belgium, in September 2010. Received June $10^{\text {th }}, 2011$. Published as resubmitted by the authors September $27^{\text {th }}, 2011$. 